\title{
Resilience in Cancer Care at the Time of COVID-19: Practical Approach to the Management of Cancer Patients During the COVID-19 Emergency in a Large Italian Community Hospital
}

\section{Angioletta Lasagna ( $\nabla$ a.lasagna@smatteo.pv.it )}

Fondazione IRCCS Policlinico San Matteo Pavia https://orcid.org/0000-0002-9611-1164

Simona Secondino

Fondazione IRCCS Policlinico San Matteo Pavia

Francesco Agustoni

Fondazione IRCCS Policlinico San Matteo Pavia

Teresa Monaco

Fondazione IRCCS Policlinico San Matteo Pavia

Ilaria Imarisio

Fondazione IRCCS Policlinico San Matteo Pavia

Anna Pagani

Fondazione IRCCS Policlinico San Matteo Pavia

Gianpiero Rizzo

Fondazione IRCCS Policlinico San Matteo Pavia

Richard J. Tancredi

Fondazione IRCCS Policlinico San Matteo Pavia

\section{Emma Pozzi}

Fondazione IRCCS Policlinico San Matteo Pavia

\section{Elisa Ferraris}

Fondazione IRCCS Policlinico San Matteo Pavia

Silvia Chiellino

Fondazione IRCCS Policlinico San Matteo Pavia

Chiara Gandini

Fondazione IRCCS Policlinico San Matteo Pavia

Silvia G. Brugnatelli

Fondazione IRCCS Policlinico San Matteo Pavia

\section{Paolo Pedrazzoli}

Fondazione IRCCS Policlinico San Matteo Pavia; University of Pavia 


\section{Method Article}

Keywords: COVID-19, cancer patients, triage, resilience, outcomes

Posted Date: August 25th, 2020

DOI: https://doi.org/10.21203/rs.3.rs-64211/v1

License: (c) (i) This work is licensed under a Creative Commons Attribution 4.0 International License. Read Full License 


\section{Abstract}

\section{Purpose}

With the emergence of coronavirus disease 2019 (COVID-19), the Oncologists have had to face the challenge of continuing active treatments without compromising the safety of our patients and healthcare personnel.

\section{Methods}

From February 24th, we reorganized our Oncology Unit with the introduction of a double-step triage strategy for cancer patients under treatment in order to identify patients at risk from COVID-19 and to avoid their admission to the outpatient clinic and to the inpatient ward.

\section{Results}

From February 24 to April 7 2020, we have performed 819 phone calls, leading to the authorization of 788 accesses (312 patients) to the outpatient clinic for active treatments. 26 patients (8.3\%) with symptoms were kept at home and managed by repeated telephone calls; 23 of them were managed at home with symptomatic treatments and antibiotics and the others 3 were hospitalized for suspected COVID. At the second triage level, 5 patients weren't admitted to the Outpatient clinic for persistent fever or respiratory distress.

177 patients were admitted to the inpatient ward: none has been found to be COVID-19 positive and both outpatient and inpatient areas were still COVID-19 free.

No healthcare workers became infected by SARS-CoV-2.

\section{Conclusion}

Our practical approach based on a simple double-step triage strategy, allows the identification of patients at risk for active COVID-19 infection, did not request neither human nor economic extra resources and appears effective, within a large community Hospital, in maintaining cancer care and therapy while protecting patients and healthcare workers from COVID-19 infection.

\section{Introduction}

Italy's COVID-19 outbreak originated in Southwest Lombardy, on February 21st, 2020. The Fondazione IRCCS Policlinico San Matteo in Pavia, the closest and largest teaching hospital near Codogno, has been involved in the management of the outbreak from the start, undergoing a rapid and thorough reorganization (Asperges et al. 2020; Perlini et al. 2020). In such an emergency, the behavior of health care workers needed to be aligned with risk management strategies, particularly to that defined as 
"resilience", a term used to define the ability to face a new situation by improving management skills (Mitchell et al. 2012). Also the oncologist community suggested to adapt the patient management to continue to assist cancer patients in the safest way, by introducing methods to improve a careful evaluation of every single patient to optimize or continuing oncological treatment (Italian Association of Medical Oncology 2020). Practically, the oncologists needed to perform a substantial quality improvement focused to avoid nosocomial COVID 19 spread. According to these suggestions, and moving from a Deming cycle (Plan - Do - Check - Act), we plan (P) and put into practice a simple and safe triage protocol (D) aimed to screen every patients before their admission into Day Hospital and Ward spaces which should remain COVID-19 free. This paper describes the obtained results (Check) to verify if and which improvements are needed $(A)$.

\section{Materials And Methods}

The outbreak of COVID-19 required an immediate reorganization of our workflow to minimize the risk of contamination. Before this emergency, there were no specific procedures to evaluate patients before admission for active treatment, nor were there any epidemiological checks with attention being paid only to signs and/or symptoms (such as fever or neutropenia) which could potentially contraindicate active therapy.

From February 24th, we choose to start a protocol based on a double-step triage strategy for cancer patients, already under treatment or newly diagnosed, consisting of:

- First step: a phone call the day before active therapy or admission

- Second step: a clinical evaluation before the admission to the outpatient and inpatient wards on the day of the treatment.

The phone call was done by an experienced clinician in order to evaluate the clinical conditions of the patient and all members of his/her family by asking about the presence of signs/symptoms as detailed in Table 1.

This assessment took account of signs and symptoms potentially related to the underlying disease or treatment toxicity.

Moreover, the clinician asked each patient if, within the previous 72 hours, he/she had been into known outbreak areas (for the first period of epidemic), or had had direct contact with people known to have been affected by COVID-19 or with people currently in quarantine. The same questions were addressed also to the patient's relatives, to identify potentially infectious close contacts.

The questionnaire was modified according to relevant information on COVID-19 published in the medical literature (e.g. after the alert on anosmia and dysgeusia as consequence of COVID-19 (Lechien et al. 2020)) and to the local protocol management (Fondazione IRCCS Policlinico San Matteo 2020). 
In the presence of symptoms potentially related to COVID-19 infection, the patient was invited to stay at home, and symptomatic treatment was suggested. Daily phone monitoring was implemented and, in cases of worsening of clinical status, the patient was reported to the general practitioner for clinical evaluation at home and eventually referred to the regional Emergency Medical System (EMS) for evaluation for hospital admission (Spina et al. 2020), according to standard protocols of outpatient management.

The second triage level was performed before the patient entered either the day hospital or the inpatient ward by the nurse case manager and a physician, both wearing personal protection equipment (PPE) as suggested by WHO guidelines (WHO 2020). This triage consisted of a new evaluation of clinical state by measuring body temperature and evaluating possible signs and symptoms of respiratory infections. This procedure was aimed at a more careful examination of patients to reinforce what emerged at the first triage level. Both in the triage area, as well as in the therapy rooms, a security distance of at least $120 \mathrm{~cm}$ was rigorously observed, and every patient was trained to wear surgical mask and shoe covers, and to disinfect, at least at admission and before discharge, the hands with an hydro-alcoholic gel. Patients who were hospital admitted, in case of fever or other suspicious symptoms, underwent a nasopharyngeal swab for SARS-CoV 2, an X-ray of the thorax and blood exams. In cases where the swab was negative, but X-ray was doubtful for a diagnosis of interstitial pneumonia, patients were not allowed to entry in the ward. With the aim of reducing social interaction, patient's relatives were not allowed to enter the hospital area.

Healthcare workers at the second level triage position and involved in the direct care of patients used the WHO-suggested PPE: eye protection (goggles), liquid-repelling gowns, double gloves, a class-2 filtering face-piece respirator (FFP2). Workers inside the Day Hospital room were equipped with surgical mask, googles, not waterproof gowns and, obviously, gloves. The aim was to supply each worker with standard protective equipment for each work-shift.

Cleaning procedures have been also implemented and standardized; in particular, ward surfaces were cleaned every day with sodium hypochloride in terminal sanitation (Colaneri et al. 2020; European Centre for Disease Prevention and Control (ECDC) (2020)). Every day, a careful check of the procedure was made, with the aim of revealing any deviation from the protocol. Furthermore, the people wearing PPE followed refresher-training sessions on their use.

\section{Results}

From February 24 to April 7 2020, we have performed 819 phone calls, leading to the authorization of 788 accesses (312 patients) to the outpatient clinic for active treatments. In the same period, one year ago, without any specific triage procedure, we recorded 820 Day hospital access. Twenty-six patients (8.3\%) with fever $\left(>37.3^{\circ} \mathrm{C}\right)$ and/or other symptoms were kept at home and managed by repeated telephone calls; of these 26 subjects, 3 were subsequently hospitalized for suspected COVID-19, while 23 were managed at home with symptomatic treatments and antibiotics. 
At the second triage level, 5 patients presented with persistent fever or respiratory distress before being admitted to the Outpatient clinic.

Overall, 5 triaged patients were hospitalized and proved positive for SARS-CoV-2 by nasal swab (figure 1).

In the same period 177 patients were admitted to the inpatient ward: 89 for cancer treatments, including 5 with germ cell tumors undergoing stem cell transplantation programs, 88 for diagnostic procedures or for supportive measures. As of April $7^{\text {th }}$, none has been found to be COVID-19-positive and both outpatient and inpatient areas were still COVID-19 free. Moreover, no healthcare workers became infected by SARSCoV-2.

The main characteristics of the patients stopped at the two triage levels are shown in the Supplementary Table 1.

\section{Discussion}

During the COVID-19 pandemic, oncologists found themselves between Scylla and Charybdis (Lewis 2020): they must protect both patients and themselves against the risk of infection and, at the same time, allow their patients to receive appropriate diagnostic work-ups and curative therapies (Tondo 2020).

So far many authors have reported the importance of organizing patient flow, including adopting strategy of telemedicine, to minimize contact between them (Hanna et al. 2020; Lambertini et al. 2020; Liu et al. 2020). The approach described in the present single-institution experience, based on a simple double-step triage strategy, allows the identification of patients at risk for active COVID-19 infection, and avoid their admission to the outpatient clinic and inpatient ward. As compared with the pre-screening protocol, we arranged our activity without substantially reducing the normal "pre-COVID" level, and without needing extra resources. During this emergency some health activities were suspended or drastically reduced, such as the follow-up visits of non-metastatic patients which represent a significant commitment "in peacetime" in terms of time worked. Also first contact visits diminished as consequence of the reduced of surgical and diagnostic procedures. Even if this kind of reduction is quite impossible to quantify, this fact has made it possible to relocate (human) resources to the triage of all patients who should have accessed the Medical Oncology and no additional resources became necessary.

Some patients were hospitalized to receive active oncological treatments or invasive diagnostic procedures because of they are frail or needed prolonged medical observation and to avoid to expose them to COVID-19 infection during their stay in various areas of the hospital.

This study has main limitations, consisting in the lack of a control and of the possibility to compare it with the approach of other Hospital in the same area: some Hospital became COVID at all and that remaining COVID free adopted completely different protocols. Moreover, we did not put in place protocols like tele-medicine but they were reserved to the management of patients remaining at home and were not enough to screen patients needing a hospital access. 
Our approach put successfully into practice the capacity to adapt our management to a global health emergency ("resilience") and to maintain high levels of safety for patients and health care workers as demonstrated by the absence of COVID-19 infections among patients and health care workers.

According to what is suggested by guidelines of improving quality in healthcare, this kind of strategy is fully sustainable even in setting were the availability of swab and serologic tests is limited. Moreover, even where diagnostic methods are available, combining a clinical and virologic approach may be useful in case of other pandemic waves when the capacity of the healthcare services may be once again overcrowded.

\section{Declarations}

\section{ACKNOWLEDGEMENTS}

We are indebted with Professor Raffaele Bruno and the medical staff of Infectious Diseases Unit for the cooperation in organizing the management strategy.

Funding: This work was partially supported by Ricerca Corrente grant no 08067619, Fondazione IRCCS Policlinico San Matteo.

Conflicts of interest: The authors declare no conflict of interest.

Code availability: Not Applicable

\section{References}

Asperges E, Novati S, Muzzi A, et al (2020) Rapid response to COVID-19 outbreak in Northern Italy: how to convert a classic infectious disease ward into a COVID-19 response centre. J Hosp Infect 105(3):477-9

Perlini S, Canevari F, Cortesi S, et al (2020) Emergency Department and Out-of-Hospital Emergency System (112-AREU 118) integrated response to Coronavirus Disease 2019 in a Northern Italy centre. Intern Emerg Med. 15(5):825-833.

Mitchell T, Harris K (2012) Resilience: A risk management approach https://www.odi.org/sites/odi.org.uk/files/odi-assets/publications-opinion-files/7552.pdf Accessed 7 April 2020

Italian Association of Medical Oncology Statement (2020) https://www.aiom.it/comunicato-aiom-peremergenza-corona-virus/ Accessed 7 April 2020

Lechien JR, Chiesta-Estomba CM, De Siati DR, et al (2020) Olfactory and gustatory dysfunctions as a clinical presentation of mild-to-moderate forms of the coronavirus disease (COVID-19): a multicenter European study. Eur Arch Otorhinolaryngol. 277(8):2251-2261. 
Fondazione IRCCS Policlinico San Matteo. COVID Management Guidelines (2020) http://intranet.sanmatteo.org/site/home/sistema-qualita/protocolli-aziendali/mcs--modellicomportamentali-standardizzati/docCatdocumenti-gestione-covid-19.1001181.1.50.1.all.html Accessed 7 April 2020.

Spina S, Marrazzo F, Migliari M, Stucchi R, Sforza A, Fumagalli R (2020) The response of Milan's emergency medical system to the COVID-19 outbreak in Italy. Lancet 395(10227):e49-e50.

WHO. Rational use of personal protective equipment (PPE) for coronavirus disease (COVID-19). https://apps.who.int/iris/bitstream/handle/10665/331498/WHO-2019-nCoV-IPCPPE_use-2020.2-eng.pdf Date: March 19, 2020. Accessed 10 April 2020)

Colaneri M, Seminari E, Piralla A, et al (2020) Lack of SARS-CoV-2 RNA environmental contamination in a tertiary referral hospital for infectious diseases in Northern Italy. J Hosp Infect 105(3):474-476.

European Centre for Disease Prevention and Control (ECDC) Technical Report. (2020) Disinfection of environment in healthcare and non healthcare settings potentially contaminated with SARS-COV-2. March 2020. Accessed 10 April 2020.

Lewis MA (2020) Between Scylla and Charybdis - Oncologic Decision Making in the Time of Covid-19. N Engl J Med 382(24):2285-2287.

Tondo L. Life 'hanging by a thread' for Italian cancer patients in coronavirus crisis. The Guardian. Tue 7 Apr 2020.

Hanna TP, Evans GA, Booth CM (2020) Cancer, COVID-19 and the precautionary principle: prioritizing treatment during a global pandemic. Nat Rev Clin Oncol. 17(5):268-270.

Lambertini M, Toss A, Passaro A, et al (2020) Cancer care during the spread of coronavirus disease 2019 (COVID-19) in Italy: young oncologists' perspective. ESMO Open 5(2):e000759.

Liu R, Sundaresan T, Reed ME, Trosman JR, Weldon CB, Kolevska T (2020) Telehealth in Oncology During the COVID-19 Outbreak: Bringing the House Call Back Virtually. JCO Oncol Pract. 16(6):289-293.

\section{Table}

Table 1: First step triage questions asked by phone to patients the day before admission and number of patients reporting signs/symptoms 


\begin{tabular}{lcc} 
Signs \& symptoms present at the time of the call or 2 weeks before & Yes & No \\
\hline Temperature $\geq 37.3^{\circ} \mathrm{C}$ & $\square$ & $\square$ \\
\hline Gastrointestinal Symptoms (nausea/vomiting/diarrhea) & $\square$ \\
\hline Respiratory symptoms & $\square$ \\
(cough/nasal discharge, dyspnea) & $\square$ \\
\hline Anosmia & $\square$ \\
\hline Ageusia & $\square$ \\
\hline Asthenia & $\square$ \\
\hline Conjunctivitis & $\square$ \\
\hline Headache & $\square$ \\
\hline Myalgia/arthralgia & $\square$ \\
\hline Patients' relatives with same symptoms & $\square$ \\
\hline
\end{tabular}

\section{Number of patients reporting symptoms (\%)}

Patients with 1 sign/symptom n. 15

Patients with 2-3 signs/symptoms n. 10

Patients with $>3$ signs/symptoms n. 1 (Fever, Cough, Anosmia)

Symptomatic relatives n. 1 (Fever)

\section{Figures}


819

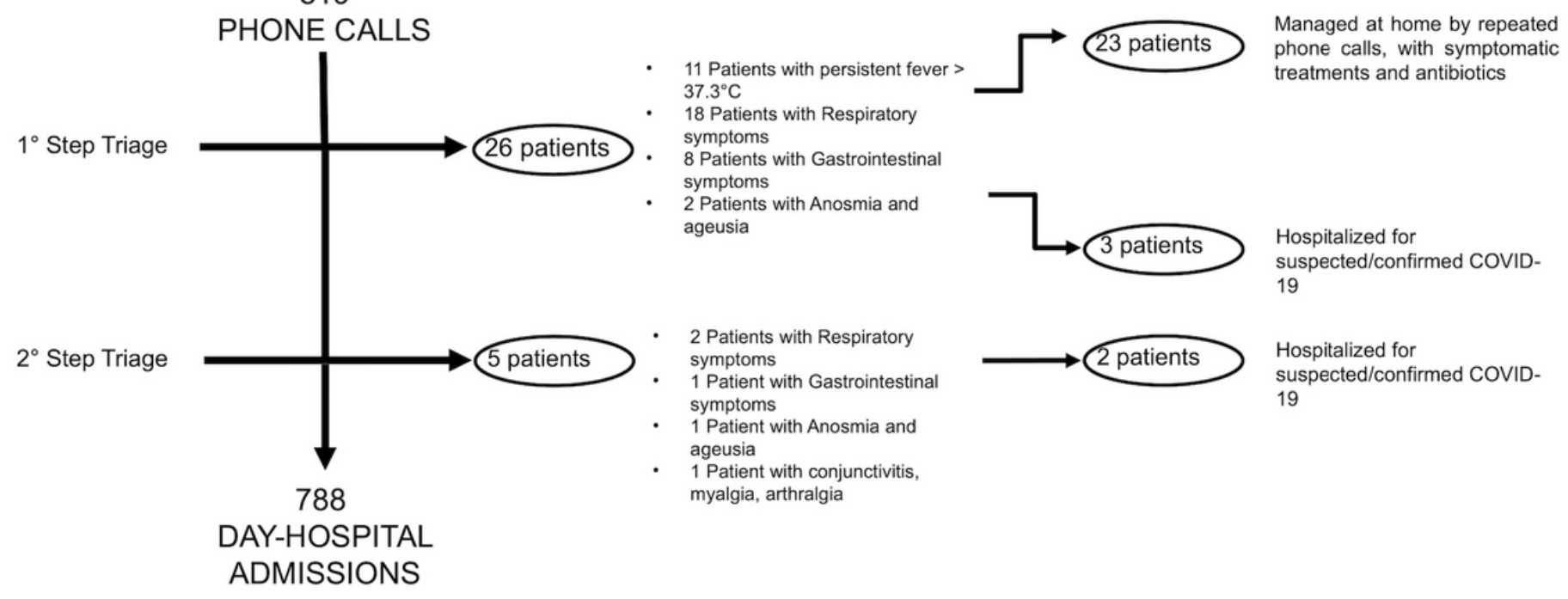

\section{Figure 1}

Outpatient management during COVID-19 emergency: a flow-chart of our double-step triage strategy.

\section{Supplementary Files}

This is a list of supplementary files associated with this preprint. Click to download.

- SupplementaryTable1.docx 